\title{
Aqueous Dispersion of Red Clay-based Ceramic Powder with the Addition of Starch
}

\author{
Maria Victoria Alcantar Umaran ${ }^{\mathrm{a} *}$, Ruben Labandera Menchavez ${ }^{\mathrm{b}}$ \\ ${ }^{a}$ Capitol University, 9000 Corrales, Cagayan de Oro City, Philippines \\ ${ }^{\mathrm{b}}$ Department of Ceramics, Chemical and Metallurgical Engineering, MSU-Iligan Institute of \\ Technology, 9200 Tibanga, Iligan City, Philippines
}

Received: August 17, 2011; Revised: October 7, 2012

\begin{abstract}
The optimum dispersion and rheological properties of red clay-based ceramic suspension loaded with unary and binary starch were investigated in aqueous medium. The aqueous ceramic suspension was prepared consisting of red clay, quartz, feldspar, and distilled water. Using a polyelectrolyte dispersant (Darvan 821A), the ternary ceramic powder was initially optimized to give the smallest average particle size at $0.8 \mathrm{wt}$. (\%) dispersant dosage as supported by sedimentation test. This resulted into an optimum high solid loading of $55 \mathrm{wt}$. (\%). The addition of either unary or binary starches to the optimized ceramic slurry increased the viscosity but maintained an acceptable fluidity. The mechanism of such viscosity increase was found to be due to an adsorption of starch granules onto ceramic surfaces causing tolerable agglomeration. Correspondingly, the rheological evaluations showed that the flow behaviors of all starch-loaded ceramic slurries can be described using Herschel-Bulkley model. The parameters from this model indicated that all ceramic slurries loaded with starch are shear thinning that is required for direct casting process.
\end{abstract}

Keywords: clay, dispersion, electrosteric, rheology, starch

\section{Introduction}

The success in achieving the desired physical properties of different types of ceramic products relies on the critical application of the colloidal ceramic processing principle $^{1}$. It involves the manipulation and control of the inter-particle forces in powder suspension in order to remove heterogeneities and to optimize the suspension properties $^{2}$. These optimum properties of powder suspension are governed by the interaction of attractive and repulsive forces. The net effect of these forces acting on particle surfaces determines the state of dispersion of the colloidal suspension wherein the repulsive forces is maximized to counteract the formation of attractive forces ${ }^{3,4}$ that cause particle agglomeration. This repulsive barrier can be achieved by electrostatic or steric stabilization mechanisms, which depends on the liquid medium and the composition of the powder.

In electrostatic stabilization mechanism, the charged particles develop an extended layer of similar charged ions in aqueous medium, which is known as the electrical double layer. The repulsion develops when there is an overlap between charged layers thus particles remain separated. As the concentration of oppositely charged ions in the aqueous medium increases, the range of the electrical double layer interaction decreases due to screening effect brought by the excess ions (oppositely charged) in the continuous medium. This ultimately causes particle agglomeration. On the other hand, the steric stabilization is effected by adsorption of polymeric additives to develop protective colloids on particle surfaces. This results into effective repulsion

*e-mail: mau_aries24@yahoo.com rendered by the extended polymer layers in the continuous liquid medium. The combination of the two mechanisms (electrostatic and steric) in dispersing the particles is called electrosteric stabilization mechanism ${ }^{1,3,7}$. Preferably, slurries or suspensions are effectively dispersed by electrosteric stabilization mechanisms ${ }^{5}$ to allow dispersion of particles at a high solid loading with minimum viscosity ${ }^{6}$.

Stable colloidal suspensions are widely employed in wet ceramic forming techniques including slip casting, tape casting, pressure casting and centrifugal casting. These techniques have been used for a long time in the forming of traditional and advanced ceramics. In those shaping techniques, the colloidal processing is an important tool in achieving the stable suspension involving the dispersion of particles that influences the succeeding forming steps. These fabrication steps include consolidation of dispersed particles by removing most of the aqueous medium, which is followed by further drying and burning out of organic additives. Subsequently, a high temperature densification is carried out to produce a final microstructure required for optimal performance ${ }^{7}$.

Several studies on the fabrication process have been carried out in a non-aqueous system with organic solvents owing to some processing advantages. However, a strong demand on the development of aqueous system is taking into account on safety, environmental issues and processing cost. Unfortunately, the use of aqueous medium presents a tendency for fine ceramic powder to agglomerate, but this can be mitigated by proper control of the surface potential through electrosteric repulsion between particles. This repulsion mechanism is conveniently provided by 
electro-steric hindrances of absorbed polymers involving polyelectrolytes to cause electrosteric stabilization ${ }^{8}$.

The solid loading also affects the dispersion behavior of the suspension ${ }^{9}$. In order to achieve high solids in ceramic suspension, this requires consideration of the processing parameters including powder characteristics, powder dispersion and wetting, slurry rheology, and introduction of shear into the slurry. A proper control of these parameters will produce a superior green microstructure with good particle packing and minimal defects such as agglomerates and pore ${ }^{10}$. Moreover, suspensions with high solid loading produce high wet and green strength that facilitate easy mold release and drying. These properties are essential in advance fabrication techniques such as gelcasting and starch consolidation, where no drainage of water takes place ${ }^{11}$.

Currently, the starch consolidation has become a competitive shaping technique owing to its environmentalfriendly process and economic viability. It offers considerable processing advantages such as easy slip casting, poreforming agents, and body forming (i.e. particle binder) agent $^{12,13}$. The starches, as slurry component, are natural biopolymer from different botanical sources including rice, corn, tapioca (cassava), wheat, and potato. The plant genotype primarily determines the size and shape of the starch granules and their properties. The size and composition of starch are key factors in determining the rheological properties of starch-containing suspensions and the viscoelastic behavior (which corresponds to the swelling and gelatinization) of starch in aqueous media at elevated temperature. In contrast to many other biopolymers and natural polysaccharides, the main advantage of using starch in ceramic technology is its chemical purity (elements $\mathrm{C}$, $\mathrm{H}$, O with only traces of other elements) that guarantees residual-free (i.e. ash-free) during burnout ${ }^{12}$. Secondary advantages are well-defined product specification (including particle size distribution), easy processing, and commercial availability $^{12,13}$. Moreover, it is used as a component and processing aid in several processes (i.e. manufacture of adhesives, textiles, paper, food, pharmaceutical and building materials) due to its thickening, gelling, adhesive and filmforming capabilities.

In fabricating porous ceramics through suspensionbased processing, the dispersion of solid particles in aqueous medium is the key issue to be addressed in order to develop a desirable porous microstructure. The starch consolidation technique is reportedly employed to produce such porous ceramics, which depends on the starch type and content in ceramic slurry mixture. In this processing technique, the dispersion behaviors of various starches mixed with pure powders including $\mathrm{Al}_{2} \mathrm{O}_{3}, \mathrm{SiC}, \mathrm{Si}_{3} \mathrm{~N}_{4}$ have been intensively reported elsewhere. However, to our knowledge, no literature has yet been published to report only on the dispersion behaviors of a clay-based powder mixture with starch addition. Particularly, a red clay-based powder mixture primarily consists of dominant amount of red clay, and additive amounts of quartz and feldspar. The clay, naturally containing iron impurity, imparts plasticity and reddish color to the powder mix while the quartz provides filler and feldspar allows low firing temperature to reduce energy requirement.

Therefore, the present study deals with the optimization of dispersion characteristics of red clay-based powder mixtures loaded with starch in aqueous medium to produce a stable and high solid loading slurry. To systematize the optimization study, the dispersion behaviors of red clay-based powder mixture without starch were firstly evaluated in terms of dispersant dosage, $\mathrm{pH}$, sedimentation, average particle size, and solid loading. Subsequently, the rheological properties of red clay-based ceramic suspension loaded with different types of starches and their binary mixtures were investigated to produce high solid loading slurry suitable for direct casting.

\section{Material and Procedure}

\subsection{Raw materials and preparation}

The red clay was mined in lumped formed from Lamalama, Lanao del Norte, Philippines. It was dried in the laboratory oven for 4 hours at a temperature of $110^{\circ} \mathrm{C}$ to completely remove the moisture. The dried lumped clay was then reduced to desired sizes using a pulverizer and was sieved passing through a 100 mesh screen. The mineral composition of the red clay according to a previous study ${ }^{14}$ is composed of montmorillonite, hematite, and alkali/alkaline bearing minerals with minor free quartz. The feldspar and quartz were used as ceramic additives and commercially supplied by Elmar Marketing, Iligan City. Correspondingly, the chemical compositions of the ceramic raw materials were given in Table 1. These materials were dispersed in distilled water by ammonium polyacrylate dispersant (Darvan 821A) with its physical and chemical properties listed in Table 2. Native starches, such as corn starch and cassava starch, were locally and commercially available, whereas a rice flour was prepared in the laboratory. To obtain the rice flour, rice grains (IR 74) were mechanically ground until a desired powder form was achieved. All powders were screened to pass through a 100-mesh screen. Subsequently, the average particle sizes (LS 100Q, Coulter Corp.) of powdered materials were presented in Table 3 for both ceramic materials and different types of starches used.

Table 1. Chemical composition of the raw materials.

\begin{tabular}{ccccccccc}
\hline \multirow{2}{*}{ Ceramic materials } & \multicolumn{8}{c}{ Oxide contents } \\
\cline { 2 - 9 } & $\mathbf{S i O}_{2}$ & $\mathbf{A l}_{2} \mathbf{O}_{3}$ & $\mathbf{F e}_{2} \mathbf{O}_{3}$ & $\mathbf{M g O}$ & $\mathbf{C a O}$ & $\mathbf{N a}_{2} \mathbf{O}$ & $\mathbf{K}_{2} \mathbf{O}$ & l.o.i. \\
\hline Red clay & 41.6 & 25.2 & 8.2 & 1.1 & 0.5 & 0.5 & 0.5 & 22.4 \\
Quartz & 95.1 & 1.7 & 0.4 & 1.4 & 0.5 & 0.1 & 0.3 & 0.6 \\
Feldspar & 66.3 & 21.5 & 0.3 & 0.5 & 2.4 & 8.5 & 0.1 & 0.4 \\
\hline
\end{tabular}


Table 2. Physical and chemical properties of dispersant.

\begin{tabular}{|c|c|c|c|}
\hline Manufacturer & R.T. Vanderbilt Co. Inc. Norwalk, CT, USA & Boiling / condensation point & $100{ }^{\circ} \mathrm{C}\left(212{ }^{\circ} \mathrm{F}\right)$ \\
\hline Commercial name & Darvan $821 \mathrm{~A}$ & Color & Yellow (light) \\
\hline Chemical name & Ammonium polyacrylate & $\mathrm{pH}$ & 7 \\
\hline Active matter (wt. (\%)) & 40 & Physical state & Liquid \\
\hline Specific gravity & 1.18 & Volatility & $60 \%(\mathrm{v} / \mathrm{v})$ \\
\hline Density & $1.16{\mathrm{~g} . \mathrm{cm}^{-3}}^{-3}\left[25^{\circ} \mathrm{C}, 77^{\circ} \mathrm{F}\right]$ & $\begin{array}{c}\text { Viscosity } \\
\text { (Brkfld \#2 Spn 30 rpm 25C, cps.) }\end{array}$ & 35 \\
\hline
\end{tabular}

Table 3. Average particle size of the raw materials.

\begin{tabular}{lccc}
\hline $\begin{array}{c}\text { Ceramic } \\
\text { materials }\end{array}$ & $\begin{array}{c}\text { Particle size } \\
(\boldsymbol{\mu m})\end{array}$ & $\begin{array}{c}\text { Types of } \\
\text { starches }\end{array}$ & $\begin{array}{c}\text { Particle size } \\
(\boldsymbol{\mu m})\end{array}$ \\
\hline Red clay & 32.34 & Corn & 13.40 \\
Quartz & 36.83 & Cassava & 15.65 \\
Feldspar & 27.99 & Rice & 43.76 \\
& & Potato & 40.92 \\
\hline
\end{tabular}

\subsection{Optimization of dispersant dosage}

The optimization was carried out with 10 wt. (\%) solid loading of the ternary powder mixture composed of $60.73 \mathrm{wt}$. (\%) clay, 22.63 wt. (\%) feldspar and 16.64 wt. (\%) quartz. This formulation was based on the stoneware specification ${ }^{15}$. After mixing the powder to its desired weight, the resulting powder mixture was added with varying concentration of 0.2 to $1.0 \mathrm{wt}$. (\%) based on the amount of ternary powder mixture. The dispersant was initially mixed with distilled water before the powder was added gradually into the liquid solution with constant stirring of the suspension. A pre-determined weight of alumina balls, which is twice the weight of the solid loading, was added into the suspension to serve as the milling media. The suspension was then ball milled altogether in polyethylene containers for 4 (four) hours with a rotation of 55 revolutions per minute (rpm) to achieve uniform dispersion of the ceramic particles. After the milling step, the average particle size was correspondingly measured against increasing amount of dispersant dosages (0.2-1.0 wt. (\%)). All measurements of average particle size were replicated at least twice to validate results and the average values were reported. A dispersant dosage that provides a lowest average particle size was decided as the optimum dispersant to render the best particle dispersion.

Using the determined optimum dispersant dosage, the sedimentation behavior of ceramic slurry at $10 \%$ solid loading was conducted at varying $\mathrm{pH}$ and compared with ceramic slurry without dispersing agent. The adjustment of the suspension $\mathrm{pH}$ was made using $\mathrm{KOH}$ and $\mathrm{HCl}$. The ceramic slurry was then transferred to graduated cylinders (100 $\mathrm{mL}$ capacity) that were covered to minimize water evaporation and allowed to stand undisturbed for 24 hours. The sediment heights were then measured directly on the graduated cylinder ${ }^{1,16,17}$.

\subsection{Solid loading optimization}

The solid loading optimization was conducted with the use of the optimum amount of dispersant determined above. The solid loadings were 45, 50, 55, 60, and $65 \mathrm{wt}$. (\%) based on the total batch weight of the ternary powder mixture. For each solid loading, the slurry was ball milled for 24 hours at $55 \mathrm{rpm}$ with predetermined weight of the alumina balls as milling media for effective deagglomeration and dispersion of ceramic particles. After this milling step, the viscosity of the slurry was measured using a viscometer (RVDE 230, Brookfield Engineering Laboratories, Inc.) equipped with spindle 4 that was rotated at varying speeds of $10,20,30$, 50, 60 and $100 \mathrm{rpm}$.

\subsection{Rheological characterization of ceramic slurry loaded with starch}

The ceramic slurry with optimum solid loading was added with 16 wt. (\%) starch based on the amount of the ternary powder. The starch concentration at this amount provided better consolidation performance ${ }^{18}$. The resulting starch-loaded ceramic slurry reduces the ternary solid loadings to $50.6 \mathrm{wt}$. (\%). Two types of such slurry were formulated and studied. The first type of the ceramic slurry was added with unary starch or single starch, whereas the second type of slurry was loaded with binary starch mixtures. All binary starch mixtures were decided to contain rice starch with a ratio of 80:20 to the other starches including potato, corn, and cassava. The starchloaded ceramic slurry was milled for 4 hours to allow homogeneous mixing of starch with the ceramic particles. Finally, rheological evaluations were then conducted with different formulations of the starch-loaded ceramic slurry.

\section{Results and Discussion}

\subsection{Dispersion behavior of red clay-based slurry without starch}

To obtain a well dispersed slurry, the particles in suspension are kept apart by a complete coverage of charged polymeric layer ${ }^{9,19}$ introduced by the optimum concentration of adsorbed polyeletrolyte. This mechanism was systematically studied for ceramic slurry containing three different types of powders including red clay, quartz, and feldspar. To determine the optimum dispersant in the range of concentrations studied, there is a concentration of dispersant at which the average particle size is at the minimum. Figure 1 shows the effect of varying concentration of dispersant on the average particle size of the ceramic slurry. It can be observed that the lowest average particle size was achieved corresponding to an optimum dispersant content of 0.8 wt. (\%) based on the amount of ternary powder. At this optimum dispersant, the $\mathrm{pH}$ is about 7.1 which is believed enough to dissociate the weak 
polyelectrolyte. If the dispersant did not dissociate, the measured average particle size may not show any trend. At this concentration, there is a complete coverage of polymeric ions on every ceramic particle to provide optimum particle dispersion as depicted by the lowest average particle size. During dispersion of clay minerals in water with such optimum dispersant, the polyacrylate anions are believed to adsorb on the positive edge of clay platelets to make the red clay particles highly negative ${ }^{20}$. On the other hand, the negatively charged dispersant adsorbs on positive sites of the minerals, such as feldspar, quartz, hematite, and their associated powders ${ }^{21}$. Hence, all surfaces of the ternary powder used in this study are possibly negative or basic to promote electrosteric repulsion.

However, in the range of dispersant concentration less than the optimum, the ceramic particles are partially covered by the polymeric ions. This results into oppositely charged ceramic particles that develop into considerable attractive forces. Ultimately, the ceramic particles are agglomerating with larger average particle size than the optimally dispersed particles. On the other hand, the addition of dispersing agent greater than the optimum concentration introduces an excess amount of polyelectrolyte in solution. This causes a compression of electrical double layers thus reducing the zeta potential to cause particle flocculation. At this state, the ceramic powder has the tendency to agglomerate due to attractive interparticle van der Waals forces. Moreover, the surfaces of the particles are saturated with dispersant molecules, and the unattached polymer molecules reside in solution to form polymer bridging that causes a decrease in the volume of the dispersing liquid ${ }^{3}$. Hence, the particles are coagulating to result into the larger average particle size than the optimally dispersed particles.

In ceramic shaping processes, (i.e. slip-casting, tape casting or spray drying), the dispersion of the ceramic powder in an aqueous medium is required. The particles in suspension should not settle fast under the action of gravity, but they should remain in suspension. Otherwise, a segregation occurs which causes density gradients in the cast objects ${ }^{22}$. The sediment height provides a valuable information about the nature of such dispersed particles. A higher sediment height with soft and loosely packed sediments will be formed for aggregated/flocculated particles. However, a stable suspension will form compact sediment of shorter height at longer time of the sediment bed formation. The compaction of such sediment is attributed to better dispersion of particles in the suspension. In contrast, a rapid formation of loose and porous sediment bed is observed for an unstable suspension ${ }^{22}$. Figure 2 illustrates the relationship between slurry $\mathrm{pH}$ and sediment height with and without the addition of optimum amount of dispersant. It can be observed that both slurries exhibit high sediment heights indicating unstability of the particle dispersion in the lower $\mathrm{pH}$ range. This unstability is attributed to an agglomeration of edge to face association of clay platelets ${ }^{23}$. The build up of such agglomerates is aggravated with the possible adsorption of quartz onto basal surfaces of clay platelets. Hence, such agglomeration causes faster sedimentation until $\mathrm{pH}$ of about 8.2 without the presence of dispersing agent. In contrast, the ceramic slurry with optimum dispersing agent demonstrated slightly higher sedimentation height until $\mathrm{pH}$ of 5.2. This is because of the fact that the polyelectrolyte

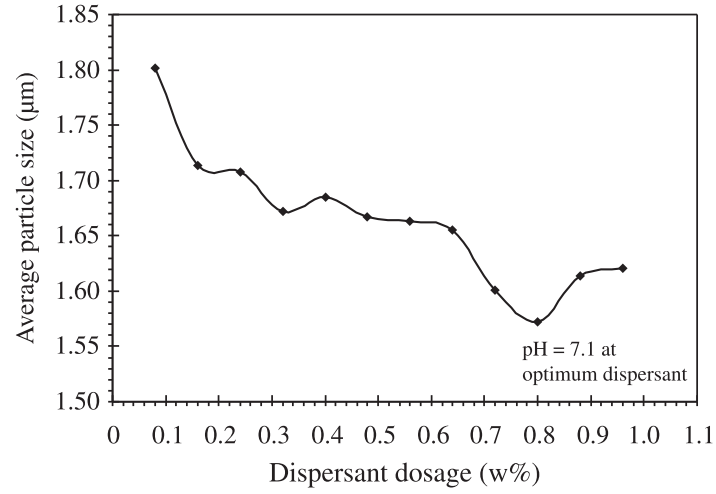

Figure 1. Effect of varying amount of dispersant on the particle size of ceramic slurry.

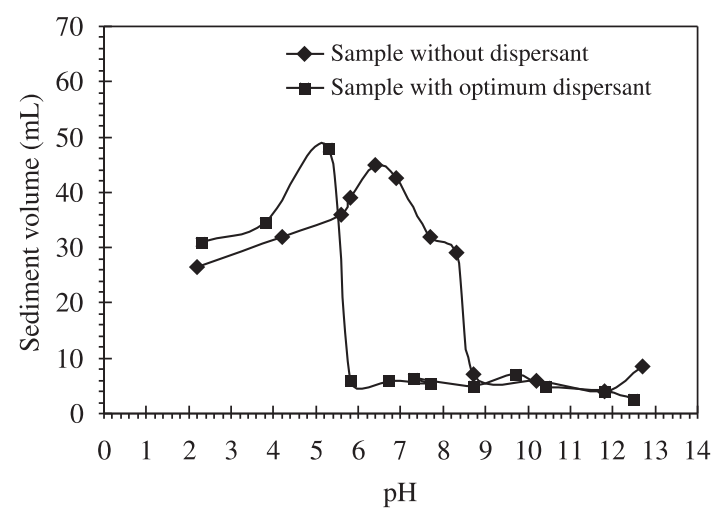

Figure 2. Sediment volume as a function of different $\mathrm{pH}$ of the ceramic slurries with and without optimum amount of dispersant.

dispersant might not dissociate at lower $\mathrm{pH}$ and favored particle clustering. Interestingly, the ceramic slurry with optimum dispersant can be dispersed at wider $\mathrm{pH}$ starting from 6 and onwards. This sedimentation data support the possible dissociation and adsorption of the dispersant onto the ceramic particles to render particle dispersion at $\mathrm{pH}$ of about 7.1. It is important to point out that the slurry without dispersant depicted a natural tendency of the particles to agglomerate and settle rapidly below the intermediate $\mathrm{pH}$. In contrast, the ceramic slurry with the addition of optimum dispersant exhibited a high degree of dispersion stability owing to the fact that particles are highly negatively charged in the presence of polymeric ions.

\subsection{Optimum high solid loading and rheological behavior of ceramic suspension}

In colloidal ceramic processing, a high solid loading is required to achieve desirable physical properties of ceramic products. This is because of the fact that the density of the ceramic slurry defines the green density of the formed ceramic material. It is therefore necessary to optimize how high the solid loading in the ceramic suspension at considerably low viscosity to allow easy casting.

Figure 3 shows the viscosity of the ceramic slurry at varying solid loadings dispersed with optimum concentration 
of dispersing agent of 0.8 wt. (\%). It can be observed that the increase in solid loading leads to an increase in the viscosity of the slurry. This could be attributed to the fact that increasing the solid loading decreases the liquid medium for dispersion causing the viscosity to approach infinity at a maximum solid fraction ${ }^{2}$. At the maximum solid loading, the adsorbed polymeric layers interlock which prevents the flow of the suspension ${ }^{24,25}$. Moreover, as the solid loading increases the separation distance between particles decreases, which causes particle agglomeration and makes the flow impossible ${ }^{22}$. For direct casting process, it is desirable that the viscosity is kept at a minimum level to allow casting. In this study, the solid loading of 55 wt. (\%) presents the optimum high solid loading with acceptable fluidity of less than 1 Pa.s at shear rate of 195/s.

The flow behavior of ceramic slurry with increasing solid loading was studied in terms of viscosity against increasing shear rate as shown in Figure 4. It can be observed that at 45 and $50 \mathrm{wt}$. (\%) solid loading the ceramic slurries behave nearly Newtonian flow. Beyond 50 wt. (\%) solid loading, the flow is apparently non-Newtonian and exhibits shear-thinning characteristics. It is worthwhile to note that shear thinning is the decrease in apparent viscosity with

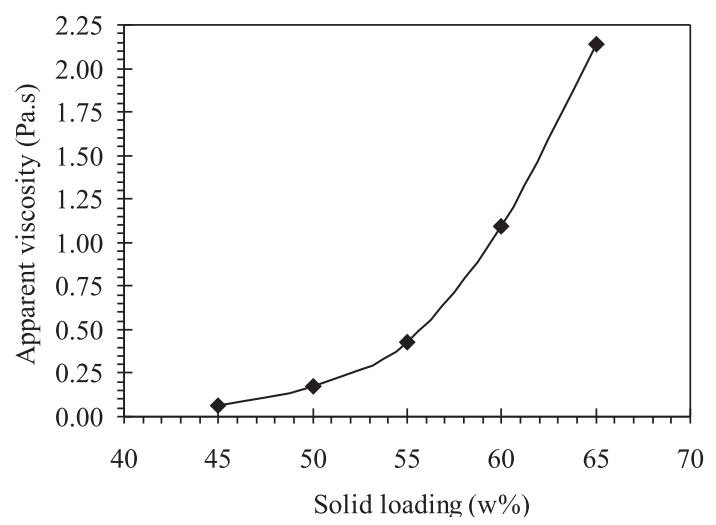

Figure 3. Apparent viscosity as a function of increasing solid loading in the ceramic slurry.

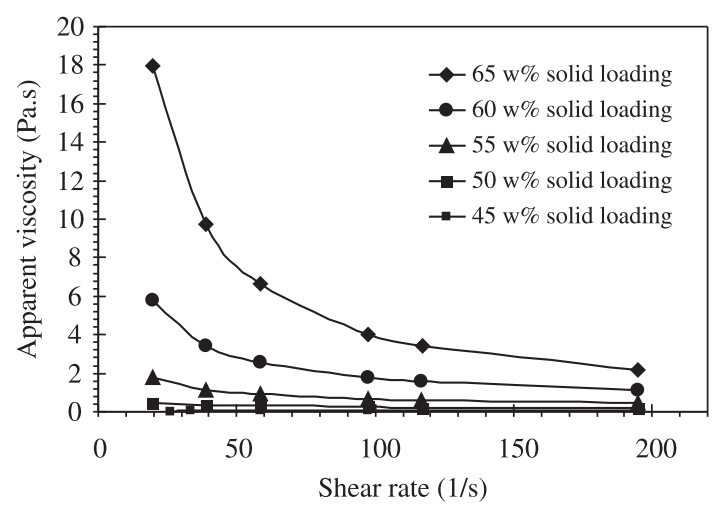

Figure 4. Viscosity versus shear rate flow curves of ceramic slurries of different percentage solid loadings. increasing shear rate. This behavior is supported with a linear trend in shear stress against increasing shear rate for all range of solid loading as depicted in Figure 5. The shear thinning behavior can be illustrated according to the arrangement of ceramic particles in suspension. During near equilibrium of ceramic suspension, random collisions among particles make them naturally resistant to flow leading to high observed viscosity. But as the shear stress (or, equivalents, the shear rate) increases, particles become organized in streamlined configuration with the flow, which ultimately lowers viscosity ${ }^{26}$.

Using the optimum high solid loading, it is important to know the rheological parameters of ceramic slurry, such as yield stress $\left(\tau_{\mathrm{o}}\right)$, power law exponent $(\mathrm{n})$, and consistency factor $(\mathrm{k})$. These parameters can be determined by fitting the collected data of increasing shear stress against shear rate (Figure 5) to existing rheological models ${ }^{27,28}$. These models are the Newtonian, Bingham, Power Law, and Herschel-Bulkley models. Table 4 lists the specific values of rheological parameters from each rheological model fitted. It is worthwhile to point out that the yield stress determines a stress at which the slurry starts to flow. Based on the highest correlation value $\left(\mathrm{R}^{2}\right)$, it was found out that the flow of ceramic slurry at $55 \mathrm{wt}$. (\%) solid loading can be described by Herschel-Bulkley model. The model indicated that the ceramic slurry started to flow at a yield stress of about $16 \mathrm{~Pa}$. The Herschel-Bulkley model can be presented by the following equation:

$\tau=\tau_{0+} k(\gamma)^{n}$

where $\tau_{0}$ is the yield stress, $\tau$ is the shear stress, $\gamma$ is the shear rate, $n$ is the power law exponent, and $k$ is a consistency factor $^{29}$.

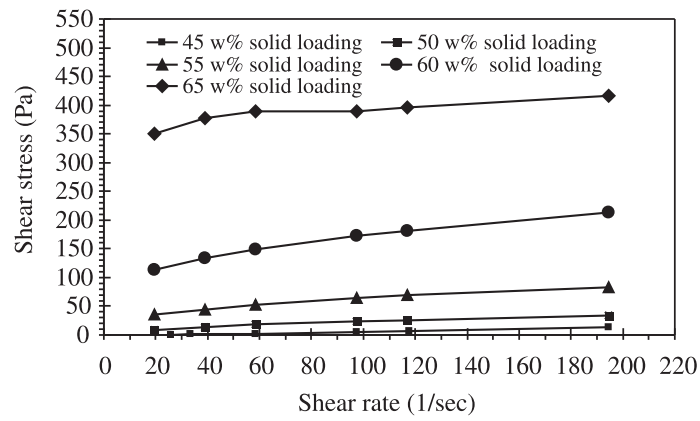

Figure 5. Shear stress versus shear rate flow curves of different percentage solid loadings.

Table 4. Fitting rheological models to optimized slurry of 55 wt. (\%) solid loading.

\begin{tabular}{ccccc}
\hline & $\begin{array}{c}\text { Newtonian } \\
\text { model }\end{array}$ & $\begin{array}{c}\text { Bingham } \\
\text { model }\end{array}$ & $\begin{array}{c}\text { Power Law } \\
\text { model }\end{array}$ & $\begin{array}{c}\text { Herschel- } \\
\text { Bulkley model }\end{array}$ \\
\hline k or m & 0.655 & 0.273 & 20.471 & 10.141 \\
$\mathrm{n}$ & 1 & 1 & 1 & 0.394 \\
$\sigma_{\mathrm{o}}$ & 0 & 48.27 & 3.019 & 16.136 \\
$\mathrm{R}^{2}$ & -1.780 & 0.981 & 0.986 & 0.991 \\
\hline
\end{tabular}




\subsection{Rheological characteristics of ceramic slurries loaded with unary and binary starch mixtures}

The starch is deliberately added to the ceramic slurry to impart binding effect between ceramic particles during consolidation step of ceramic slurry. In this study, the amount of starch was $16 \%$ based on ternary solid loading that reduced the ceramic loading to about $50.6 \mathrm{wt}$. (\%). Since the success of the consolidation step depends upon uniform binding of particles through thermal dissolution of starch, the added starch particles need to be homogeneously mixed in ceramic suspension. This is usually achieved through the addition of the optimum dispersant to ceramic slurry containing starch. Hence, Table 5 presents a variation of average particle size and $\mathrm{pH}$ against increasing dispersant dosage based on $10 \mathrm{wt}$. (\%) solid loading. It can be observed that the optimum dispersant dosage is maintained at 0.8 wt. $(\%)$. This indicates that the dispersant preferentially adsorbed onto ceramic particles rather than onto starch granules $^{21,23}$. This is because the starch granules might electrostatically reject the dispersant due to its partially negative surface at the $\mathrm{pH}$ studied $^{30}$. Moreover, there is a need to understand the dispersion behavior of ceramic particles at high solid loading in the presence of starch.

When starch was added to ceramic slurry, the available liquid for particle dispersion was reduced due to the concentration and the associated liquid wetting of starch granules. These contributed to the increase in viscosity when compared to ceramic slurry without starch. The viscosity will continue to significantly increase depending on the surface charge of the ceramic particles, average particle size and polymeric constituents of starch used. Because of these parameters, the viscosity of ceramic slurry should be prepared at minimum to give allowance for possible increase in viscosity. Table 6 lists the viscosity of ceramic slurry as a function of starch types and average particle size. It can be observed that the viscosity of starch-loaded ceramic slurry increases with increasing average particle size. The increased average particle size is the indication of particle flocculation/ agglomeration due to possible starch adsorption. Moreover, the viscosity increases in the following order of starch used: corn $<$ cassava $<$ rice $<$ potato. This trend of viscosity increase could be attributed to the interaction of dispersantcoated ceramic surfaces and the starch granules during dispersion. Since all surfaces of the ternary powder were negatively modified by dispersant and the starch granules were reported to behave positively charged particles, the adsorption of such granules onto the ceramic particles was very likely during wet mixing ${ }^{30}$. This starch adsorption causes an inevitable formation of large particle clusters that hinder the flow of particles past each other leading to the increased viscosity. In fact, there is a large increase in viscosity of ceramic slurry loaded with cassava starch when compared to ceramic slurry alone as listed in Table 6 . The observed viscosity is aggravated when potato starch was loaded to ceramic slurry. It is believed that the highest viscosity of potato-loaded ceramic slurry was probably due to the polymeric constituents including amylopectin and amylose. The amylose is a linear molecule containing lipids whereas the amylopectin is a branched polymer associated with phosphate groups. According to literatures ${ }^{31,32}$, the concentration of polymeric constituents varies with botanical source and the amylopectin content follows in increasing trend of corn, rice, potato, and cassava. It is reported that the amylopectin polymer has a strong tendency to attach on basic surfaces than amylose ${ }^{33}$. Although the cassava starch has the highest content of amylopectin, the synergistic interaction with amylose could possibly lower the build up of particle clustering leading to the viscosity reduction. In the case of rice starch, the highest viscosity

Table 5. Optimization of dispersing agent in the presence of starch particles (10 wt. (\%) ternary powder +16 wt. $(\%)$ starch based on ternary powder).

\begin{tabular}{cccccccccc}
\hline \multirow{2}{*}{$\begin{array}{c}\text { Dispersant } \\
\text { dosage (wt. }(\%))\end{array}$} & \multicolumn{3}{c}{ pH of starch-loaded ceramic slurry } & \multicolumn{5}{c}{ Average particle size $(\boldsymbol{\mu m})$} \\
\cline { 2 - 9 } & corn & cassava & rice & potato & corn & cassava & rice & potato \\
\hline 0.6 & 6.6 & 6.7 & 6.9 & 6.8 & 1.541 & 1.578 & 15.21 & 1.530 \\
0.8 & 6.7 & 6.9 & 7.0 & 6.7 & 1.514 & 1.540 & 14.33 & 1.493 \\
1.0 & 6.8 & 7.0 & 7.1 & 6.8 & 1.540 & 1.548 & 16.51 & 1.509 \\
1.2 & 6.9 & 7.1 & 7.2 & 6.9 & 1.566 & 1.550 & 17.27 & 1.559 \\
\hline
\end{tabular}

Highlighted values in italicized format are average particle size of starch-loaded ceramic slurry at optimum dispersant dosage.

Table 6. Relationship of viscosity of starch-loaded ceramic slurries to starch types and mean particle size and starch.

\begin{tabular}{|c|c|c|c|}
\hline \multirow{2}{*}{$\begin{array}{c}\text { Starch } \\
\text { type }\end{array}$} & \multirow{2}{*}{$\begin{array}{c}\begin{array}{c}\text { Pure } \\
\text { starch }\end{array} \\
\text { Particle size } \mu \mathrm{m} \text { (ave) }\end{array}$} & \multicolumn{2}{|c|}{$\begin{array}{l}\text { Ceramic slurry (55 wt. }(\%)) \\
\text { with } 16 \text { wt. }(\%) \text { starch }\end{array}$} \\
\hline & & $\begin{array}{l}\text { Particle size } \\
\mu \mathrm{m} \text { (ave) }\end{array}$ & $\begin{array}{c}\text { Viscosity } \\
195 \mathrm{~s}^{-1} \text { (Pa.s) }\end{array}$ \\
\hline Corn & 13.40 & 1.681 & 0.784 \\
\hline Cassava & 15.65 & 1.695 & 1.006 \\
\hline Potato & 40.92 & 1.684 & 1.394 \\
\hline Rice & 43.76 & 1.759 & 1.342 \\
\hline Starch-free ceramic slurry & \multicolumn{2}{|c|}{ Particle size, $\mu \mathrm{m}: 1.675$} & 0.430 \\
\hline
\end{tabular}


could be due to the largest particle agglomerations despite of poorer amylopectin content. Lastly, the ceramic slurry loaded with corn starch demonstrated the lowest viscosity among the starch-loaded ceramic slurries. This is because it has the smallest average size of particle agglomeration.

In principle, the viscosity of the suspension decreases with increasing particle size. However, based on our findings above, the trend is opposite (Table 6). This is possibly attributed to the agglomeration/clustering of ceramic and starch particles as discussed above ${ }^{34}$. The starch adsorption onto ceramic surfaces is also aggravated by the presence of iron in clay as hematite mineral to cause flocculation. This is because of the fact that the amylopectin in starch had a greater affinity of adsorption onto hematite surface ${ }^{35}$. To verify this claim, the average particle size of starch-loaded ceramic slurries was measured at varying time as shown in Table 7. It can be observed that all of the starch-loaded ceramic slurries showed increasing average particle size as indication of particle agglomeration. However, the percentage of particle size agglomeration is decreasing against increasing viscosity. This is maybe because larger particles have lesser tendency towards adsorption than smaller ones. To further confirm the agglomeration, starch slurries with addition of dispersing agent based on the ternary powders were studied at varying time as presented in Table 8. It can be seen that the average particle size is increasing along with $\mathrm{pH}$ until 8.3 as an indication of particle flocculation. The average particle size of starch slurries is increasing except for rice starch slurry. It is believed that the rice granules were reduced into fine size during milling and facilitated adsorption onto ceramic particles resulting into the largest agglomeration size. Therefore, the information on milling pointed out that the mixing of starch-based ceramic slurry should not be carried out longer than four hours to avoid increasing viscosity.

The rheological characteristics of the optimized ceramic slurry (55 wt. (\%) solid loading) added with 16 wt. (\%) unary starch and binary starches (based on the amount of ceramic powder) were evaluated. It is worthwhile to note that the addition of $16 \mathrm{wt}$. (\%) starch based on the ternary powder reduces the ceramic loading to about $50.6 \mathrm{wt}$. (\%). The different types of starches studied were corn, cassava, rice, and potato. Figures 6 and 8 depict the viscosity of all starch-loaded ceramic slurries against increasing shear rate. It can be observed that all ceramic slurries loaded either unary or binary mixture showed the same trend of decreasing viscosity against increasing shear rate. This slurry characteristic is an indication of the shear thinning behavior suitable for direct casting. Specifically, all starchloaded ceramic slurries demonstrated a good fluidity with viscosity around $1 \mathrm{~Pa} . \mathrm{sec}$ at shear rate of 195/s. Moreover, the observed shear thinning property can be attributed to the breakdown of the structures of particle networks created by the inter-particle forces and particle orientation effects under the action of applied shear stress.

The corresponding rheological parameters of all starchloaded ceramic slurries were determined using the stress and shear rate data as shown Figures 7 and 9. It can be seen that all slurries exhibit a linear trend in terms of shear

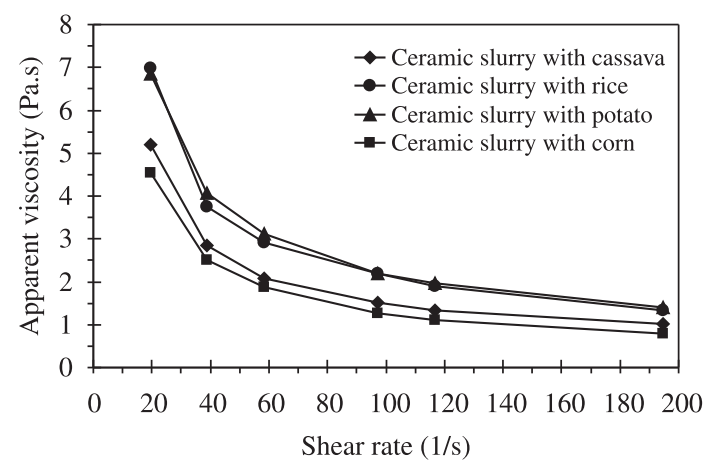

Figure 6. Viscosity versus shear rate flow curves for the single starch-loaded ceramic slurries.

Table 7. Mean particle size agglomeration (in micrometers) of the optimized ceramic slurry loaded with different types of starch at increasing milling time.

\begin{tabular}{lccccc}
\hline \multirow{2}{*}{$\begin{array}{c}\text { Ceramic slurry added } \\
\text { with starch }\end{array}$} & \multicolumn{5}{c}{ Milling time (hours) } \\
\cline { 2 - 6 } & $\mathbf{1}$ & $\mathbf{2}$ & $\mathbf{3}$ & $\mathbf{4}$ & \% size agglomeration \\
\hline Corn & 1.618 & 1.687 & 1.694 & 1.666 & 2.97 \\
Cassava & 1.681 & 1.683 & 1.688 & 1.695 & 0.83 \\
Rice & 1.730 & 1.743 & 1.756 & 1.759 & 1.68 \\
Potato & 1.672 & 1.673 & 1.675 & 1.684 & 0.72 \\
\hline
\end{tabular}

\%particle agglomeration $=((\mathrm{P} 4-\mathrm{P} 1) / \mathrm{P} 1)^{*} 100$, where $\mathrm{P} 4$ is average particle size at 4 hours and $\mathrm{P} 1$ is average particle size at 1 hour.

Table 8. Effect of milling time on the $\mathrm{pH}$ and average particle size of pure starch slurries treated with optimum dispersant.

\begin{tabular}{|c|c|c|c|c|c|c|c|c|}
\hline \multirow{2}{*}{$\begin{array}{l}\text { Milling time } \\
\text { (hours) }\end{array}$} & \multicolumn{4}{|c|}{ pH } & \multicolumn{4}{|c|}{ Average particle size $(\mu \mathrm{m})$} \\
\hline & corn & cassava & rice & potato & corn & cassava & rice & potato \\
\hline 1 & 7.7 & 7.7 & 7.4 & 7.5 & 14.09 & 17.12 & 25.07 & 54.22 \\
\hline 2 & 7.9 & 7.9 & 7.6 & 7.7 & 16.56 & 20.42 & 18.42 & 63.04 \\
\hline 3 & 8.0 & 8.0 & 7.8 & 7.9 & 17.61 & 22.04 & 13.59 & 64.93 \\
\hline 4 & 8.3 & 8.3 & 8.1 & 8.1 & 17.50 & 22.43 & 11.62 & 69.56 \\
\hline
\end{tabular}




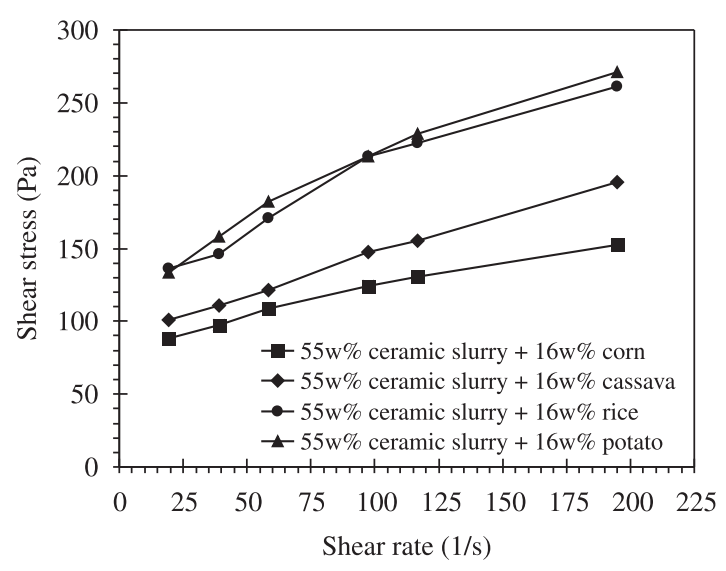

Figure 7. Shear stress versus shear rate flow curves for single starch-loaded ceramic slurries.

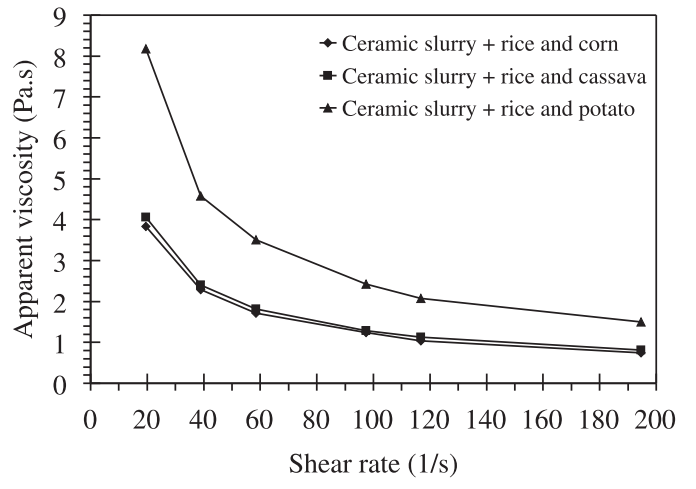

Figure 8. Viscosity versus shear rate flow curves for the binary starch-loaded ceramic slurries.

Table 9. Herschel-Bulkley parameters of single starch-loaded slurries.

\begin{tabular}{lccccc}
\hline $\begin{array}{c}\text { Slurry } \\
\text { added with starch }\end{array}$ & $\begin{array}{c}\text { Viscosity } \\
\mathbf{1 9 5 ~ s}^{\mathbf{- 1}}(\mathbf{P a . s})\end{array}$ & $\begin{array}{c}\text { Yield stress } \\
\sigma_{\mathbf{o}}(\mathbf{P a})\end{array}$ & $\begin{array}{c}\text { Consistency } \\
(\mathbf{k})\end{array}$ & $\begin{array}{c}\text { Power Law exponent } \\
(\mathbf{n})\end{array}$ & $\begin{array}{c}\text { Correlation } \\
\left(\mathbf{R}^{\mathbf{2}}\right)\end{array}$ \\
\hline Corn & 0.784 & 29.534 & 20.042 & 0.341 & 0.987 \\
Cassava & 1.006 & 36.074 & 15.17 & 0.44 & 0.990 \\
Rice & 1.342 & 44.642 & 23.650 & 0.422 & 0.967 \\
Potato & 1.394 & 41.846 & 26.435 & 0.410 & 0.998 \\
\hline
\end{tabular}

Table 10. Herschel-Bulkley parameters of binary starch-loaded slurries.

\begin{tabular}{lccccc}
\hline $\begin{array}{c}\text { Slurry } \\
\text { added with } \\
\text { binary starch }\end{array}$ & $\begin{array}{c}\text { Viscosity } \\
\mathbf{1 9 5} \mathbf{~ s}^{-\mathbf{1}}(\mathbf{P a . s})\end{array}$ & $\begin{array}{c}\text { Yield stress } \\
\sigma_{\mathbf{0}} \mathbf{( P a )}\end{array}$ & $\begin{array}{c}\text { Consistency } \\
(\mathbf{k})\end{array}$ & $\begin{array}{c}\text { Power Law exponent } \\
(\mathbf{n})\end{array}$ & $\begin{array}{c}\text { Correlation } \\
\left(\mathbf{R}^{\mathbf{2}}\right)\end{array}$ \\
\hline Rice-Corn & 0.75 & 19.960 & 18.313 & 0.365 & 0.996 \\
Rice-Cassava & 0.814 & 25.884 & 15.853 & 0.400 & 0.997 \\
Rice-Potato & 1.498 & 51.308 & 35.656 & 0.358 & 0.989 \\
\hline
\end{tabular}

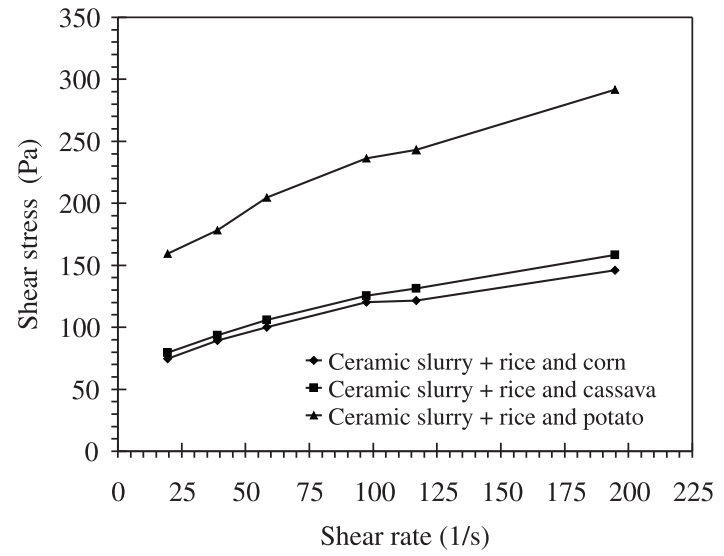

Figure 9. Shear stress versus shear rate flow curves for the binary starch-loaded ceramic slurries.

stress and increasing shear rate. These data were fitted to rheological models mentioned in section 3.2. The resulting fitted parameters were presented in Tables 9 and 10 for both ceramic slurries loaded with unary starch and binary starch mixtures. Based on the highest statistical correlation value
$\left(\mathrm{R}^{2}\right)$, the flow behavior of all starch-loaded ceramic slurries can be described using Herschel-Bulkley model. The model indicated all values of power law exponent (n) to be less than 1 . According to literature ${ }^{29}$, any values of $n<1$ indicate shear thinning whereas $n>1$ shows that the slurry is shear thickening. Based on these conditions, all slurries evaluated in this study (Tables 4, 9 and 10) exhibited shear thinning that is required for direct casting process. It is further observed that the yield stress of starch-loaded ceramic slurries is higher than the yield stress of ceramic slurry without starch. This indicates that higher stress is required to initiate the flow, but in this study the fluidity of all starchloaded slurries is low enough for slurry processing. The yield stress of starch-loaded ceramic slurry increases according to the order of starch type used: corn $<$ cassava $<$ potato $<$ rice. This observed trend could be related to the degree of agglomeration in ceramic slurry. In the case of ceramic slurry loaded with binary starch mixture, the flow behavior can also be described by the Herschel-Bulkley model as shown in Table 10. The yield stress is only the highest for rice-potato mixture, but the viscosity is low enough for slurry processing at about $1 \mathrm{~Pa}$.sec. This observable flow behavior is expected since the mixture is involved with starches that elevate the slurry viscosity. Inspite of it, according to the 
power law exponent the ceramic slurry loaded with binary starch mixture is shear thinning as supported with the plot of viscosity against increasing shear rate (Figure 8). The significance of using the binary starch mixture is simply to alter or supplement the less performing starch during dispersion and consolidation step. This study disclosed that any starch that imparts high viscosity to ceramic slurry could be diluted with low viscosity starch to reduce the final slurry viscosity. However, when both starches are individually imparting high viscosity to ceramic slurry the final viscosity will be higher as listed in Table 9 and 10 .

\section{Conclusion}

The optimum dispersion characteristics and rheological properties of red clay-based ceramic suspension loaded with different types of starches and their binary mixtures were successfully achieved in producing stable and high solid loading slurry. This was realized by effecting the electrosteric stabilization mechanism on ceramic particles with the addition of optimized amount of 0.8 wt. (\%) polyelectrolyte dispersant in aqueous medium. This resulted into optimum high solid loading of $55 \mathrm{wt}$. (\%). The addition of different types of starch and their binary mixtures to such optimized ceramic slurry increased the viscosity around $1 \mathrm{~Pa}$.s but it was low enough for slurry processing.

\section{References}

1. Briscoe BJ, Khan AU and Luckham PF. Optimizing the Dispersion on an Alumina Suspension Using Commercial Polyvalent Electrolyte Dispersants. Journal of the European Ceramic Society. 1998; 18(14):2141-2147. Particle Tech. Group, Imperial Col. Sci. Tech. Med. London, P11:SO955-2219.

2. Bergstrom L. Colloidal Processing of Ceramics. In: Holmberg K. Handbook of Applied Surface and Colloid Chemistry. John Wiley \& Sons Ltd.; 2001.

3. Bao Y, Senos MR, Almeida M and Gauckler LJ. Rheological behavior of aqueous suspensions of hydroxyapatite (HAP). Journal of Materials Science: Materials in Medicine. 2002; 13:639-643. PMid:15348572. http://dx.doi. org/10.1023/A:1015773322821

4. Hidber PC, Graule TJ and Gauckler LJ. Influence of the Dispersant Structure on Properties of Electrostatically Stabilized Aqueous Alumina Suspensions. Journal of the European Ceramic Society. 1997; 17:239-249. http://dx.doi. org/10.1016/S0955-2219(96)00151-3

5. Adachi N, Hashiba M and Sakurada O. Rheological properties of slurries prepared using planetary mixer. Ceramics International. 2004; 30:1055-1058. http://dx.doi.org/10.1016/j. ceramint.2003.11.008

6. Zhang Q, Li W, Gu M and Yanping J. Dispersion and rheological properties of concentrated silicon aqueous suspension. Powder Technology. 2006; 161:130-134. http://dx.doi.org/10.1016/j. powtec.2005.10.005

7. Shqau K. Electrosteric dispersants used in colloidal processing of ceramics. Columbus: Group Inorganic Materials Science, Department Materials Science \& Engineering, Ohio State University; 2005. p. 1-16. (Literature review).

8. Sakamoto W, Nakaoka H, Yogo T and Hirano S. Dispersability of $\mathrm{BaTiO}_{3}$ Aqueous Slurries with Poly Ammonium Acrylate Based Dispersant. Journal of the Ceramic Society of
The mechanism of such viscosity increase was found to be due to the adsorption of starch granules onto ceramic surfaces causing tolerable agglomeration. Correspondingly, the rheological evaluations showed that the flow behaviors of all starch-loaded ceramic slurries can be described using Herschel-Bulkley model. The parameters from this model indicated that all ceramic slurries loaded with starch are shear thinning that is suitable for direct casting process. Furthermore, the optimum conditions of the red clay-based slurry obtained in this study can be set as baseline data for any ceramic suspension loaded with starch.

\section{Acknowledgements}

The authors gratefully acknowledge a financial support from Commission on Higher Education (PGMA-CHED Scholarship) and Capitol University, Cagayan de Oro City. Thanks are also due to the College of Engineering (ChE/ CerE/MetE Lab) of MSU-Iligan Institute of Technology for allowing us to use any equipment during the conduct of this work. The authors would also like to thank R.T. Vanderbilt Company Inc., USA for free supply of the dispersing agent (Darvan 821A). Furthermore, we would like to extend our sincere thanks to the reviewers of this manuscript for giving us valuable comments to make our paper scientifically relevant.

Japan. 2003; 111:811-814. http://dx.doi.org/10.2109/ jcersj.111.811

9. Li X and Li Q. YAG ceramic processed by slip casting via aqueous slurries. Ceramics International. 2008; 34:397-401. http://dx.doi.org/10.1016/j.ceramint.2006.10.018

10. Janney MA. Attaining High Solids in Ceramic Slurries. Oak Ridge: Metals and Ceramics Division, Oak Ridge National Laboratory. TN 37831. Available from: <http://www.ornl. gov>. Access in: 01/2010.

11. Palmqvist L, Lyckfeldt O, Carlstrom E, Davoust P, Kauppi A and Holmberg K. Dispersion mechanisms in aqueous alumina suspensions at high solids loadings. Colloids and Surfaces A: Physicochemical and Engineering Aspects. 2006; 274:100-109. http://dx.doi.org/10.1016/j.colsurfa.2005.08.039

12. Gregorova E and Pabst W. Process control and optimized preparation of porous alumina ceramics by starch consolidation casting. Journal of the European Ceramic Society. 2011; 31:2073-2081. http://dx.doi.org/10.1016/j. jeurceramsoc.2011.05.018

13. Gregorova E, Pabst W, Zivcova Z, Sedlarova I and Holikova S. Porous alumina ceramics prepared by wheat flour. Journal of the European Ceramic Society. 2010; 30(14):2871-2880. http:// dx.doi.org/10.1016/j.jeurceramsoc.2010.03.020

14. Ochotorena ZL. Chemical analysis of clay resources of Iligan City and Lanao del Norte. The Technician. 1986; (5):11-38.

15. Menchavez RL and Intong L. Red clay-based porous ceramic with pores created by yeast-based foaming technique. Journal of Materials Science. 2010; 45:6511-6520. http://dx.doi. org/10.1007/s10853-010-4740-9

16. Singh BP, Menchavez RL, Takai C, Fuji M and Takahashi M. Stability of dispersions of colloidal alumina particles in aqueous suspensions. Journal of Colloid and Interface Science. 2005; 291:181-186. PMid:15964586. http://dx.doi. org/10.1016/j.jcis.2005.04.091 
17. Tseng WJ and $\mathrm{Wu} \mathrm{CH}$. Sedimentation, rheology and particle packing structure of aqueous $\mathrm{Al}_{2} \mathrm{O}_{3}$ suspensions. Ceramics International. 2003; 29:821-828. http://dx.doi.org/10.1016/ S0272-8842(03)00023-3

18. Pacut GA and Tumilap LC. Fabricaion of Red Clay-Based Porous Ceramic through Yeast-Based Foaming with Different Variety of Rice Flours. [Thesis]. Mindanao Midwestern State University-Iligan Institute of Technology; 2011.

19. Tsetsekou A, Agrafiotis C and Milias A. Optimization of the rheological properties of alumina slurries for ceramic processing applications Part I: Slip-casting. Journal of the European Ceramic Society. 2001; 21:363-373. http://dx.doi. org/10.1016/S0955-2219(00)00185-0

20. Tateyama H, Scales PJ, Ooi M, Nishimura Rees KS and Healy TW. X-ray Diffraction and Rheology Study of Highly Ordered Clay Platelet Alignment in Aqueous Solutions of Sodium Tripolyphosphate. Langmuir. 1997; 13:2440-2446. http:// dx.doi.org/10.1021/la960995s

21. Ibrahim I, Hussin H, Azizli KAM and Alimon MM. A study on the interaction of feldspar and quartz with mixed anionic/cationic collector. Journal of Fundamental Sciences. 2011; 7:101-107.

22. Greenwood R, Rowson N, Kingman S and Brown G. A new method for determining the optimum dispersant concentration in aqueous grinding. Powder Technology. 2002; 123:199-207. http://dx.doi.org/10.1016/S0032-5910(01)00457-0

23. Barany S, Meszaros, Marcinova L and Skvarla J. Effect of polyelectrolyte mixtures on the electrokinetic potential and kinetics of flocculation of clay mineral particles. Colloids and Surfaces A: Physicochemical and Engineering Aspects. 2011; 383:48-55. http://dx.doi.org/10.1016/j. colsurfa.2011.01.051

24. Shin Y, Su CC and Shen YH. Dispersion of aqueous nanosized alumina suspensions using cationic polyelectrolyte. Materials Research Bulletin. 2006; 41:1964-1971. http:// dx.doi.org/10.1016/j.materresbull.2006.01.032

25. Singh BP, Bhattacharjee S, Besra L and Sengupta DK. Comparison between techniques based in charge characterization and capillary suction time for assessing the dispersion characteristics of concentrated slurry. Journal of Materials Science. 2004; 39:2437-2442. http://dx.doi. org/10.1023/B:JMSC.0000020007.83933.1f

26. Cheng X, McCoy JH, Israelachvili JN and Cohen I. Imaging the microscopic structure of shear thinning and thickening colloidal suspensions. Science, 2011; 333:1276-1279. PMid:21885778. http://dx.doi.org/10.1126/science.1207032

27. Binner JGP and McDermott AM. Rheological characterization of ammonium polyacrylate dispersed concentrated alumina suspension. Ceramics International. 2006; 32:803-810. http:// dx.doi.org/10.1016/j.ceramint.2005.06.004

28. Menchavez RL. Electrical and Physical Properties of Dense and Porous Alumina with In-Situ Nano-Carbon Network Fabricated by Combination of Gelcasting and Reduction Sintering. [Dissertation]. Japan: Nagoya Institute of Technology; 2008.

29. Dimitri FD, Verhoeven R and De Schutter D. Evaluation of Time Independent Rheological Models Applicable to Fresh SelfCompacting Concrete. Applied Rheology. 2007; 17(5):562441-56244-10.

30. Laskowski JS, Liu Q and O'Connor CT. Current understanding of the mechanism of polysaccharide adsorption at the mineral/ aqueous solution interface. International Journal of Mineral Processing. 2007; 84:59-68.

31. Srichuwong S, Sunarti TC, Mishima T, Isono N and Hisamatsu M. Starches from different botanical sources II: Contribution of starch structure to swelling and pasting properties. Carbohydrate Polymers. 2005; 62:25-34. http://dx.doi. org/10.1016/j.carbpol.2005.07.003

32. Charles AL, Chang YH, Ko WC, Sriroth K and Huang TC. Influence of Amylopectin Structure and Amylose Content on the Gelling Properties of Five Cultivars of Cassava Starches. Journal of Agricultural and Food Chemistry. 2005; 53:27172725. PMid:15796616. http://dx.doi.org/10.1021/jf048376+

33. Liu Q, Zhang Y and Laskowski JS. The adsorption of polysaccharides onto mineral surfaces: an acid/base interaction. International Journal of Mineral Processing. 2000; 60:229245. http://dx.doi.org/10.1016/S0301-7516(00)00018-1

34. Weissenborn PK, Warren LJ and Dunn JG. Selective flocculation of ultrafine iron ore, I. Mechanism of adsorption of starch onto hematite. Colloids and Surfaces A: Physicochemical and Engineering Aspects. 1995A; 99:11-27. http://dx.doi. org/10.1016/0927-7757(95)03111-P

35. Weissenborn PK. Behaviour of amylopectin and amylose components of starch in the selective flocculation of ultrafine iron ore. International Journal of Mineral Processing. 1996; 47:197-211. http://dx.doi.org/10.1016/03017516(95)00096-8 